\title{
Unificación de enseñanzas relacionadas con el Tratamiento Digital de Señales en la Universitat de València*
}

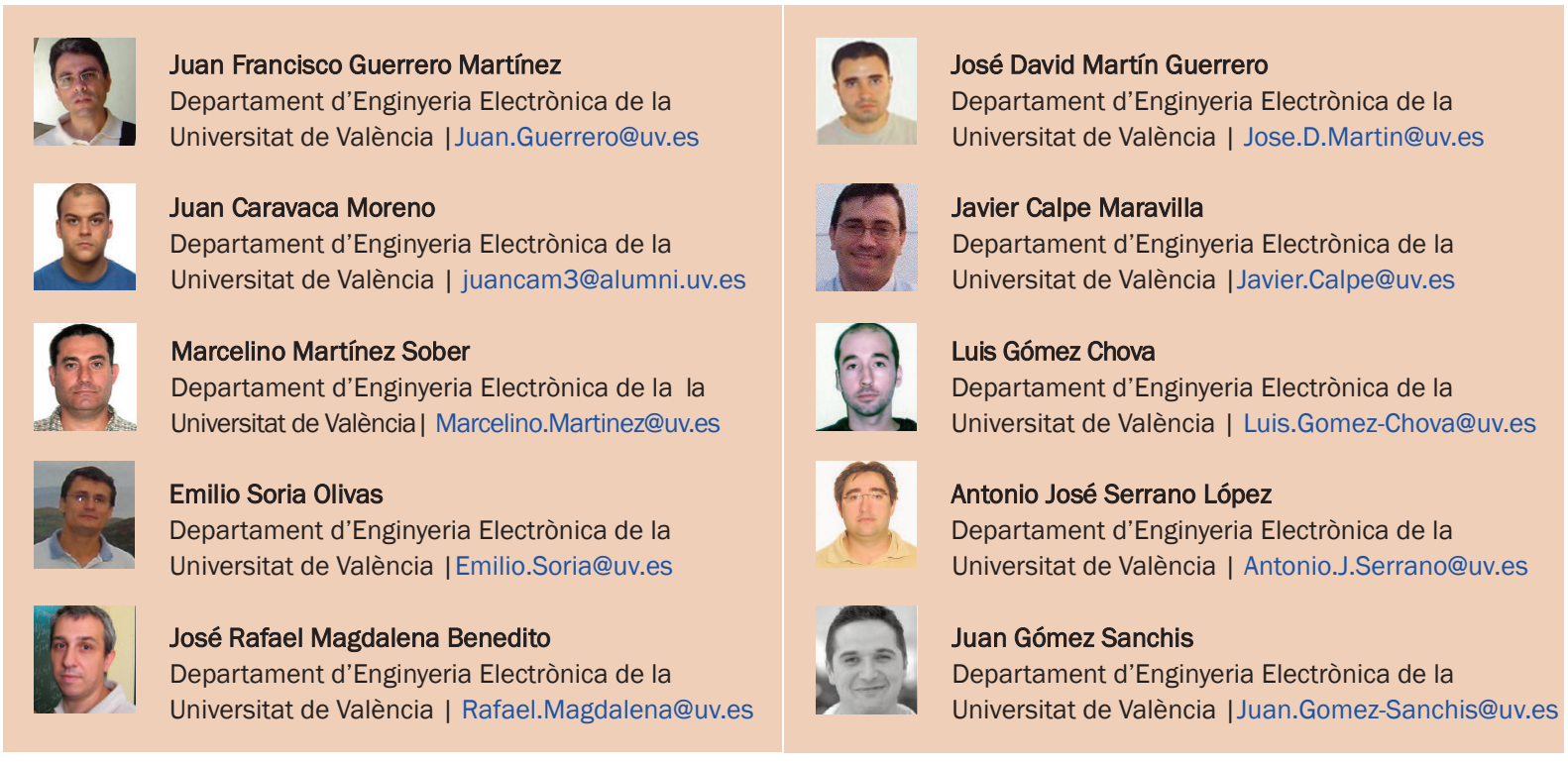

|Fecha presentación: 26/09/2010 | Aceptación: 01/04/2011 |Publicación: 21/06/2011

Resumen

La docencia del Tratamiento Digital de Señales (TDS) se realiza en diversos módulos, con una gran intersección de contenidos. En el presente trabajo se desarrolla una estrategia, que permite la unificación de contenidos de TDS, pudiéndose extraer los correspondientes a cada asignatura que componen el mapa curricular de TDS. Además, se plantea que el repositorio común de los contenidos se base en una plataforma para gestión de recursos, analizando en este trabajo el caso de Moodle y DotLRN. La solución final consiste en un repositorio en formato HTML formado por unidades básicas. Estas unidades básicas se agrupan en paquetes estándar IMS-CP, formando los materiales de las distintas asignaturas.

Palabras clave: Tratamiento Digital de Señales, aprendizaje en línea, DotLRN, Moodle, Aula Virtual, IMS-CP

\section{Resum}

La docència del Tractament Digital de Senyals (TDS) es realitza en diversos mòduls, amb una gran intersecció de continguts. Este treball planteja el desvolupament d'una estratègia que permeta la unificació de continguts de TDS, de manera que es puguen extraure els corresponents a cada assignatura que composen el mapa curricular de TDS. Amés, es planteja que el repositori comú dels continguts es base en una plataforma per gestió de recursos, tot analitzant el cas de Moodle i DotLRN. La solució final consistix en un repositori en format HTML format per unitats bàsiques. Estes unitats bàsiques s'agrupen en paquets estàndard IMS-CP, formant els materials de les diferents assignatures.

Paraules clau: Tractament Digital de Senyals, aprenentatge en línia, DotLRN, Moodle, Aula Virtual, IMS-CP

* Proyecto DocenTIC de la Universitat de València (convocatoria 2009-2010): “Unificación de enseñanzas relacionadas con el Tratamiento Digital de Señales en la Universitat de València", Ref. 32/DT/13. 


\begin{abstract}
Teaching Digital Signal Processing (DSP) at the University of Valencia takes place in several subjects with a great overlap in contents. This work proposes an approach to unify DSP contents, so that it is possible to extract the contents corresponding with each subject. Moreover, the proposal is based on a repository that contains the common contents; the repository is hosted on a virtual learning environment, being Moodle and DotLRN the two platforms under study. The final implemented solution consists in an HTMLrepository formed by a number of basic teaching units. These basic units are packaged in a standard IMS-CP format, making up the materials of the different subjects.
\end{abstract}

Key words: Digital Signal Processing, e-learning, DotLRN, Moodle, Aula Virtual, IMS-CP

\section{Introducción}

La docencia de la teoría y aplicaciones del Tratamiento Digital de Señales (TDS) se realiza en asignaturas de diversas titulaciones. Dentro de la Universitat de València (UV), que es donde se ha desarrollado esta experiencia, aparece esta temática tanto en los antiguos planes a extinguir como en los grados y másteres que están comenzando a implantarse: Ingeniería Técnica de Telecomunicaciones, especialidad Sistemas Electrónicos (ITTSE), Ingeniería Técnica de Telecomunicaciones, especialidad Telemática (ITTT), Ingeniería Electrónica (IE), Máster Interuniversitario de Ingeniería Biomédica (MIB), Máster Interuniversitario de Ingeniería Ambiental (MIA), programas de doctorado de Ingeniería Electrónica (PDIE) e Informática y Matemática Computacional (PDIMC), Grado de Ingeniero Electrónico de Telecomunicaciones (GIET), Grado de Ingeniero en Electrónica Industrial (GIEI) y Máster en Ingeniería Electrónica (MIE).

Dentro de estas titulaciones, existen diversas asignaturas vinculadas al TDS en la totalidad o parte de su programa. Suman un total de 22, incluyendo troncales y optativas, así como cursos de doctorado. Están impartidas por un grupo de profesores que desarrollan los programas y recursos docentes propios de su asignatura concreta. Dichos profesores pertenecen al Departamento de Ingeniería Electrónica (DIE) de la UV, lo que facilita las tareas de coordinación de contenidos. Esta tarea de coordinación se hace, fundamentalmente, por titulaciones, intentando obtener una trayectoria curricular en TDS coherente para el alumno, sin duplicidades ni lagunas en contenidos básicos.

No obstante, puesto que el cuerpo de conocimiento de TDS es unitario, existe también un alto grado de intersección entre las diferentes asignaturas. Esto implica en la práctica que los profesores que desarrollan sus materiales docentes para cada asignatura particular no aprovechan, en la mayoría de los casos, los recursos ya elaborados por otros docentes.

El objetivo principal del presente proyecto es desarrollar un repositorio común de contenidos, en el campo de la enseñanza del TDS, que permita una unificación de los mismos y del que se pueda extraer los contenidos necesarios para cada asignatura en particular. Esto tendría como ventaja una optimización del uso de recursos previamente elaborados. Además, facilitaría en gran medida las tareas de coordinación entre asignaturas, así como el diseño de trayectorias curriculares coherentes en las titulaciones en que se imparten y el desarrollo de nuevas asignaturas asociadas a los nuevos planes de estudio: en la bibliografía se pueden encontrar algunos trabajos relacionados. En Dharmadhikari et al. (2010) se plantea un curso de introducción al TDS realizado bajo la plataforma Moodle, y en YingPing et al. (2007) se describe un curso que permite la adaptación, en cierta medida, a los conocimientos o al modo de aprendizaje elegido por el alumno. En Santos et al. (2007a: 170) se describe el desarrollo de un entorno de aprendizaje en línea basado en estándares educativos bajo la plataforma dotLRN, planteando una metodología de creación de cursos.

El grupo de innovación educativa Grupo de Procesado Digital de Señal (GID-GPDS) de la UV está formado por la práctica totalidad de los profesores que imparten docencia en las asignaturas vinculadas al TDS comentadas anteriormente. Dichos profesores tienen una experiencia de más de 10 años en este tipo de docencia, y han desarrollado los programas y recursos docentes de dichas asignaturas. Basándose en esta experiencia, los objetivos concretos del presente trabajo son:

- Realizar un repositorio de contenidos de TDS que abarque de forma transversal las distintas asignaturas que forman el proyecto.

- Organizar dichos contenidos en un módulo de teoría.

- Desarrollar los materiales docentes en formatos compatibles con sistemas de gestión de recursos de aprendizaje (LMS: Learning Management System). En este caso, se analizará especialmente DotLRN por ser el utilizado en Aula Virtual (AV), que es la plataforma de aprendizaje en línea de la UV.

A continuación, se describe la metodología y resultados obtenidos en la realización del módulo de teoría, dentro del proyecto DocenTIC 2009-10, aprobado por el Vicerrectorado de Planificación e Igualdad de la UV

\section{Métodos}

El desarrollo del proyecto se organizó en las siguientes etapas:

1. Recopilación de información sobre: a) materiales docentes actualmente disponibles para las diversas asignaturas; b) herramientas para la implementación del proyecto; c) AV: análisis de posibilidades y limitaciones. 2. Implementación del módulo de teoría: a) análisis y unificación de formatos; b) desarrollo de los submódulos del repositorio; c) desarrollo de manuales de uso.

3. Evaluación del sistema implementado: a) pruebas piloto parciales en las distintas fases de desarrollo del módulo; b) desarrollo de cuestionarios; c) análisis de opiniones de los usuarios finales.

En los siguientes apartados se describe la implementación de dichas etapas.

2.1 Recopilación de información

En primer lugar, se elaboró un listado de los materiales docentes previamente desarrollados en las asignaturas incluidas en el presente proyecto. Dichos materiales estaban accesibles por parte del alumno en $\mathrm{AV}$ o en páginas web específicas de las asignaturas. Algunos tipos de materiales, como guías docentes o presentaciones teóricas en el aula, estaban presentes en todos los casos. No obstante, otros, como apuntes teóricos, boletines de problemas, exámenes 
resueltos o materiales de prácticas, tenían una representación muy variable en función de cada asignatura. Además, los formatos eran distintos, aunque predominaban $p d f$, $d o c$ (Word) o ppt (Power Point).

$\mathrm{El}$ siguiente paso fue analizar las diferentes opciones de implementación del proyecto, que contemplaba la generación de un módulo de teoría que recogiera los contenidos de las asignaturas relacionadas con el TDS impartidas dentro de la UV. Era necesario desarrollar un temario común que surgiría de la fusión de los distintos materiales de dichas asignaturas. A partir de estos contenidos generales, se extraerían los específicos para cada asignatura concreta. Este concepto abierto permite generar contenidos específicos no sólo para asignaturas existentes sino también para futuras.

Para implementar el acceso al contenido de las asignaturas se planteó la utilización de herramientas $L M S$, ya que permiten aprovechar el entorno de usuario de dicha plataforma, por lo que se puede centrar los esfuerzos en el desarrollo de los contenidos propios. El alumno, al entrar en la plataforma e identificarse, accedería solamente a los materiales de la asignatura que está cursando. En esta fase, se analizaron dos de las plataformas más utilizadas: Moodle y DotLRN (Santos et al. 2007b: 195).

Moodle presenta características interesantes para el desarrollo del presente proyecto (Al-Aljan et al. 2008). Tiene una estructura muy flexible, permitiendo incorporar módulos que añaden nuevas funcionalidades. Además, posibilita la creación de un repositorio de contenidos comunes con vinculación a distintos cursos, que es uno de los puntos de partida del proyecto. Para ello, es necesario definir las unidades de aprendizaje en línea mediante el estándar IMS-CP (IMS Content Packaging), utilizado en diversos LMS y que consiste en la descripción mediante XML de paquetes de páginas HTML que siguen una estructura jerárquica. Al tratarse de un estándar, IMS-CP asegura que los objetos de aprendizaje desarrollados para el repositorio dispondrán de interoperabilidad para poder ser utilizados en distintas plataformas (Santos et al. 2007a: 170;Latorre et al. 2009). Como ventaja adicional, y muy importante en este contexto, Moodle admite la edición de fórmulas en formato TeX (Waud et al. 2003).

La plataforma AV también permite incluir contenidos definidos por paquetes $I M S-C P$. Sin embargo, en contraposición con $\operatorname{DotLRN}$, en el cual se basa, AV no admite utilizar un repositorio desde el cual se vinculen los mate-

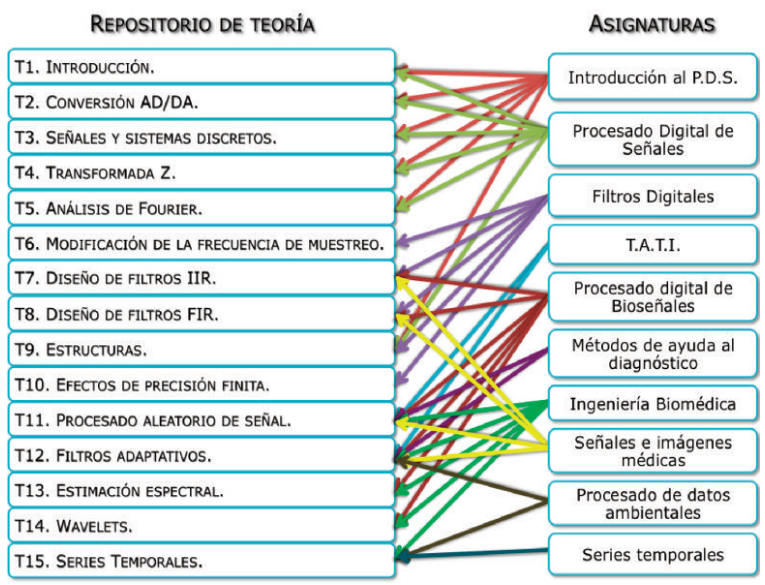

Figura 1. Correspondencias entre los contenidos del módulo de teoría (izquierda) y de las diversas asignaturas (derecha). riales de las distintas asignaturas del proyecto de forma dinámica (Pastor et al. 2009). Para cada curso se ha de desarrollar un paquete IMS-CP específico, con los submódulos básicos del repositorio general que le correspondan. Como características adicionales que presenta la plataforma $\mathrm{AV}$ y que, por tanto, son aplicables para el presente proyecto, se tiene:

- Control de acceso (sólo usuarios matriculados en las asignaturas correspondientes).

- Almacenamiento de materiales docentes para su descarga por los alumnos (módulo Recursos).

- Autoevaluación de conocimientos (módulo Cuestionarios).

- Comunicación entre usuarios (noticias, foro, chat).

- Posibilidad de escribir fórmulas en formato TeX.

La decisión final fue adoptar AV ya que permite integrar el proyecto en una plataforma utilizada por la UV, lo que asegura una autenticación conforme a la utilizada por el resto de servicios de la universidad, así como una mayor familiarización con su utilización por parte de los usuarios finales.

2.2. Implementación del módulo de teoría

En primer lugar se, determinó la estructura del repositorio, con el objetivo de cubrir de forma sistemática los contenidos de TDS. Se elaboró un programa genérico del módulo de teoría, acordado por todos los profesores que imparten asignaturas de TDS, analizándose las correspondencias entre el material disponible y dicho programa (Figura 1). El material de cada tema se organizó en submódulos básicos, para conseguir generar las diferentes combinaciones de contenidos considerados necesarios para cada asignatura.

La siguiente fase, unificación de formatos de los contenidos, estaba muy ligada a la elección de la plataforma que permitiría el acceso a los mismos. En esta fase, se diseñaron diversas pruebas piloto utilizando un mismo tema teórico con diversos formatos: pdf, flash, html. Los dos primeros se descartaron ya que dificultaban la definición de contenidos en submódulos básicos, así como las actualizaciones de los mismos. El formato HTML facilita la integración de diapositivas y apuntes, permitiendo además la edición del módulo de teoría de una forma sencilla, por lo que se decidió implementar los submódulos básicos en este formato.

A continuación se desarrollaron los contenidos del módulo de teoría en ficheros $H T M L$, cada uno de los cuales define un submódulo básico. Estos ficheros hacen uso de hojas de estilo en cascada (CSS) y Javascript para mostrar u ocultar contenidos de ampliación. Además, las fórmulas están generadas mediante formulación TeX. Se elaboraron los paquetes $I M S-C P$ de las distintas asignaturas a partir de los contenidos del repositorio, según las correspondencias definidas en la Figura 1.

Una vez desarrollado el módulo, su funcionamiento se probó mediante la generación de una comunidad dentro de $\mathrm{AV}$, donde se cargaron los paquetes generados. Los contenidos finales de cada asignatura fueron revisados por los profesores responsables de las mismas, para detectar posibles errores y redundancias en los módulos.

Debido al concepto abierto del módulo de teoría, que puede incorporar nuevas asignaturas y ser utilizado por diferentes profesores, se desarrollaron también diversos manuales para facilitar la futura ampliación y edición del temario general, así como la creación de nuevos paquetes $I M S-C P$. Esta documentación incluye: 
a) Manual y plantilla para la creación de contenidos $H T M L$ siguiendo los estilos utilizados en el temario general.

b) Manual para la creación de nuevos paquetes $I M S-C P$ a partir de los contenidos HTML del temario general; c) Manual para incorporar paquetes $I M S-C P$ a un curso de Aula Virtual.

\subsection{Evaluación del sistema implementado}

Durante el desarrollo del módulo de teoría se fueron realizando pruebas parciales en diferentes etapas, como se comentó en el apartado anterior. Otro tipo de evaluación está orientada al alumno que accede a los contenidos de su asignatura cuyo objetivo final es determinar su grado de comprensión de los mismos. Para ello, se realizaron dos cuestionarios de prueba utilizando el propio interfaz de AV para cuestionarios y usando la herramienta comercial Respondus. El cuestionario realizado con Respondus se generó de forma local y después se cargó en AV. Se observó que esta herramienta no presentaba ventajas significativas respecto a utilizar directamente el módulo de cuestionarios de $\mathrm{AV}$, y además necesita licencia, por lo que se descartó su uso.

Por último, para analizar las opiniones de los usuarios finales (alumnos) se desarrolló un cuestionario específico. Se seleccionó el grupo que se examinaba en la convocatoria de junio de 2010 de la asignatura Introducción al Procesado Digital de Señales de $2^{\circ}$ curso de la ITTSE. Para ello se generó su paquete $I M S-C P$ y se cargó en el correspondiente curso de AV. Se preparó también un cuestionario en forma

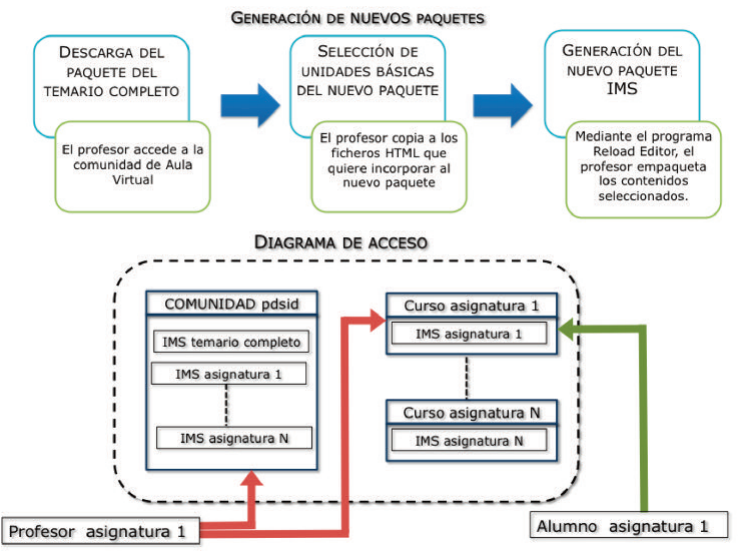

Figura 2. Diagrama de la integración del módulo de teoría y los paquetes IMS de cada asignatura, así como el acceso por parte de profesor y alumno.

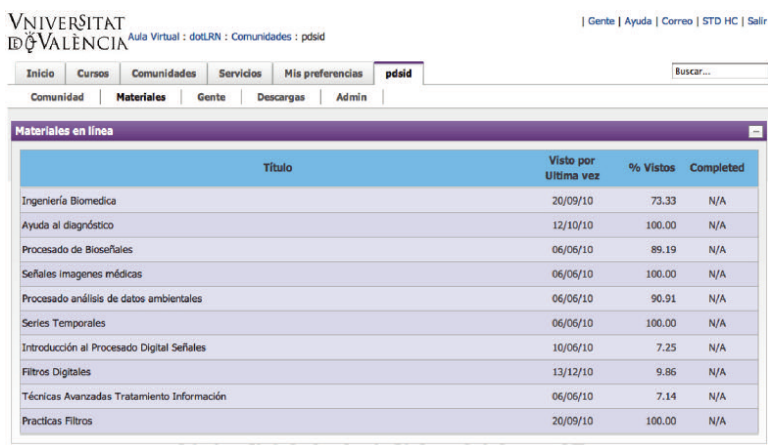

Figura 3a. Repositorio de paquetes creados.
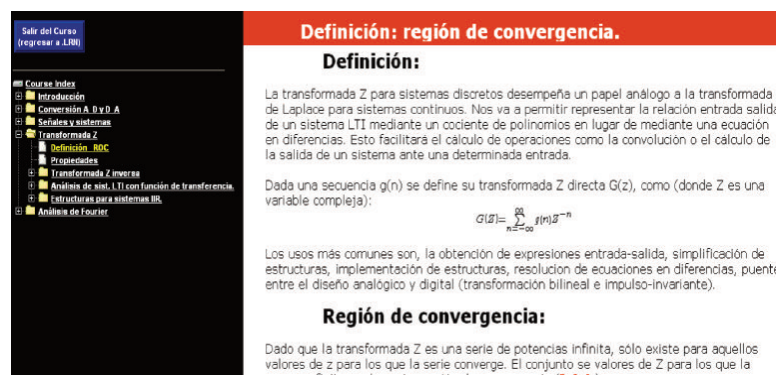

Figura 3b. Ejemplo de presentación de contenidos para el usuario final. de encuesta (Anexo 1) para que los alumnos valorasen los distintos aspectos del módulo de teoría, y poder realizar la evaluación. Las ocho primeras preguntas del cuestionario están basadas en el documento Learning Object Review Instrument (LORI) (Nesbit et al. 2003) que permite realizar una evaluación sobre los aspectos fundamentales del aprendizaje en línea (Anexo 2).

\section{Resultados}

La utilización de la plataforma Aula Virtual implica la creación de una comunidad en la que están alojados los paquetes $I M S-C P$ de todas las asignaturas, además de un paquete que engloba todo el temario. Esta comunidad sirve de enlace entre los distintos cursos de forma que cada administrador de curso puede descargar el paquete de su asignatura y subirlo a su curso, desde donde puede acceder el alumno (Figura 2).

La Figura 3 (a y b) muestra el acceso a los distintos paquetes, creados a partir del repositorio general, desde la comunidad PDSID (a), así como un ejemplo de contenidos (correspondientes a la asignatura IPDS) tal y como se presentan al alumno (b).

La evaluación de los resultados del proyecto por parte de los usuarios finales fue satisfactoria, aunque limitada debido a que por plazos de desarrollo sólo pudo hacerse con un pequeño número de alumnos (17 encuestas). En el apéndice A.1 se muestran las preguntas y resultados del cuestionario. Cabe destacar que, aunque los resultados son muy satisfactorios, deben ampliarse a un mayor número de usuarios para asegurar su validez.

Se ha desarrollado una página web del grupo de innovación docente (http://haddock.uv.es/gpds/index.php/es/docencia/grupo-de-innovacion-educativa) donde se recoge información sobre el presente proyecto y otras actividades del grupo. Además, la zona privada de dicha página permite a los miembros del grupo compartir los contenidos docentes desarrollados.

\section{Conclusiones}

El presente trabajo describe la implementación de un concepto de repositorio genérico de material docente que permite unificar contenidos de diferentes asignaturas relacionadas con TDS. El concepto modular adoptado permite generar fácilmente material para nuevas asignaturas mediante diversas combinaciones de los submódulos básicos, lo que le permite adaptarse a las asignaturas propuestas y a otras futuras. Esta aproximación puede generalizarse para cubrir no sólo los contenidos de TDS, sino también otro tipo de contenidos incluidos en las asignaturas. Para ello, basta con añadir al programa general los submódulos específicos necesarios hasta abarcar la totalidad del temario. Por la misma razón, su extensión a otro tipo de materias distintas del TDS es totalmente factible. 


\section{Bibliografía}

Al-Ajlan, Ajlan; Zedan, Hussein (2008). Why Moodle. En 12th IEEE International Workshop on Future Trends of Distributed Computing Systems, Kunming: IEEE Computer Society, 2008, pp.58-64.

Dharmadhikari, V. B; Loni, D.Y. (2010). DSP Course Teaching Using Moodle. En International Conference on Signal Acquisition and Processing. Bangalore: IEEE Computer Society, pp.268-273.

Latorre, Martin et al. (2009). Reutilización de objetos educativos para estudio de circuitos electrónicos. En IEEE Revista Iberoamericana de Tecnologías del Aprendizaje pp.37-44 .Vol. 4, Núm. 1.

Nesbit, John; Belfer, Karen; Leacock, Tracey (2003). Learning Object Review Instrument (LORI). E-Learning Research and Assessment Network, http://www.elera.net/eLera/Home/Articles/LORI\%2 01.5.pdf. Fecha de consulta 28/02/2011.

Pastor, Rafael et al. (2009). Los recursos tecnológicos para la teleformación en España: Comparativa e implantación. En IEEE Revista Iberoamericana de Tecnologías del Aprendizaje, Vol. 4, Núm. 1. pp.27-34. http://romulo.det.uvigo.es/revista/RITA/site/200902/uploads/IEEE-RITA.2009.V4.N1.A5.pdf
Respondus (2010). Características técnicas de Respondus 4.o. http://www.respondus.com/products/respondus.shtml. Fecha de consulta 28/02/2011.

Santos, O. C. et al. (2007a). Cursos accesibles y reusables sobre la plataforma ALPE. Proceedings of the FLOSS (Free/Libre/Open Source Systems) International Conference 2007. Jerez de la Frontera, pp. 170-185.

Santos, O. C. (2007b). Why using dotLRN? UNED use cases,.Proceedings of the FLOSS (Free/Libre/Open Source Systems) International Conference 2007. Jerez de la Frontera, pp. 195-212.

Universitat de València (2010). Guía de uso para el Aula virtual. http://aulavirtual.uv.es/global/HELP. Fecha de consulta 28/02/2011.

Waud, Douglas /2003). What is TeX? TUGboat, volume 24, number 2, pp.163-164. http://www.tug.org/TUGboat/Articles/tb24-2/tb77waud-what.pdf

YingPing, Hu; Yong, Lian (2007). An adaptive E-learning portal for DSP learning. En 6th International Conference on Information, Communications \& Signal Processing. Singapore: IEEE Computer Society, pp.1-5.

\section{| Cita recomendada de este artículo}

Guerrero Martínez, Juan F.; Caravaca Moreno, Juan; Martínez Sober, Marcelino; Soria Olivas, Emilio; Magdalena Benedito, José Rafael; Martín Guerrero, José David; Calpe Maravilla, Javier; Gómez Chova, Luis; Serrano López, Antonio J. y Gómez Sanchis, Juan (2011). Unificación de enseñanzas relacionadas con el Tratamiento Digital de Señales en la Universitat de València. @tic. revista d'innovació educativa. ( $\left.n^{\circ} 6\right)$. URL. Fecha de consulta, dd/mm/aaaa. 


\section{Anexo 1. Encuesta: Introducción al Procesado Digital de Señales}

Evaluación de los recursos de Introducción al Procesado Digital de Señales para el aprendizaje online. Las preguntas se puntuarán utilizando una escala del 1 (bajo) al 5 (alto), excepto las preguntas 9 y 10. Si la pregunta no es relevante para la evaluación del objeto de aprendizaje o si el evaluador no se siente capacitado para juzgarla, puede marcar NA (No Aplica).

1. Calidad de los contenidos. (Veracidad, exactitud, presentación equilibrada de ideas y nivel adecuado de detalle)

2. Motivación. (Capacidad de motivar y generar interés en un grupo concreto de alumnos/as)

3. Diseño y presentación. (El diseño de la información audiovisual favorece el adecuado procesamiento de la información)

4. Usabilidad. (Facilidad de navegación, interfaz predictiva para el usuario y calidad de los recursos de ayuda de la interfaz)

5. Utilidad de la nueva presentación de contenidos con respecto a los materiales impresos utilizados habitualmente 6. ¿Recomendaría esta presentación de materiales para el uso extensivo en otras asignaturas?

7. ¿Considera que sería interesante la creación de formularios para la evaluación online de cada módulo?

8. Esta nueva presentación de contenidos facilita el aprendizaje del módulo teórico de la asignatura.

9. ¿Qué porcentaje de la nota total del módulo asignaría a la evaluación continua?

10. ¿Qué porcentaje de la nota total del módulo asignaría al examen?

La siguiente Gráfica 1 muestra los resultados obtenidos para las 8 primeras preguntas:

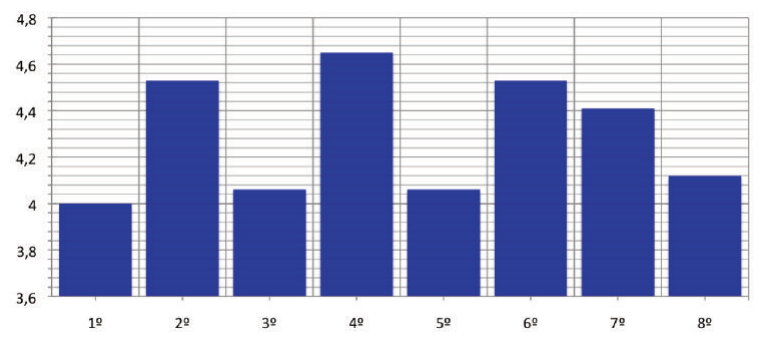

Gráfica 1. Valores medios y desviaciones estándar de cada una de las 8 primeras preguntas incluidas en el cuestionario.
Anexo 2. Instrumento para la evaluación de objetos de aprendizaje (LORI). Hoja de puntuación

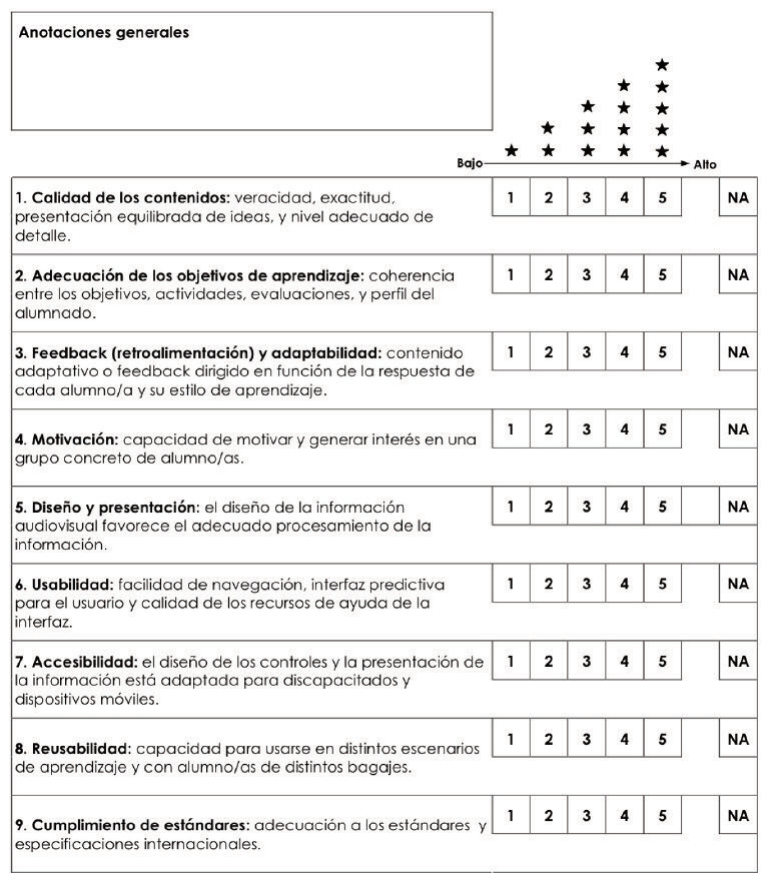

